\title{
Effect of alogliptin on hypertensive chronic kidney disease patients with type 2
}

\section{diabetes mellitus}

Amira Said ${ }^{1}$, Nadia Hussain², Amal Hussain Ibrahim Al Haddad³, and Farideh Javid ${ }^{4}$

1. Department of Clinical Pharmacy, College of Pharmacy, Al Ain University of Science and Technology, Al Ain, Abu Dhabi, UAE

2. Department of Pharmaceutical Sciences, College of Pharmacy, Al Ain University of Science and

Technology, Al Ain, Abu Dhabi, UAE

3. Institute of Public Health, College of Medicine and Health Sciences, UAE University, Al Ain, UAE

4. Department of Pharmacy, School of Applied Sciences, University of Huddersfield, Queensgate,

Huddersfield, United Kingdom

\section{RESEARCH}

Please cite this paper as: Said A, Hussain N, Al Haddad AHI, Javid F. Effects of Alogliptin on hypertensive chronic kidney disease with Type 2 diabetes mellitus. AMJ 2018;11(2):113123. https://doi.org/10.21767/AMJ.2018.3319

\section{Corresponding Author:}

Dr. Amira SA Said

Department of Clinical Pharmacy, College of Pharmacy

Al Ain University of Science and Technology

Al Ain, Abu Dhabi, UAE

P.O.Box: 64141, UAE

Email: amira.ahmed@aau.ac.ae

\section{ABSTRACT}

\section{Background}

Diabetes mellitus (DM) is a leading cause of chronic kidney disease (CKD). The antihyperglycemic treatment options for patients with Type $2 \mathrm{DM}$ are limited because of safety and tolerability concerns.

\section{Aims}

To retrospectively assess the effect of using Alogliptin; a dipeptidyl peptidase-4 inhibitor (DPP-4i) along with conventional gliclazide: a sulphonylurea (SU) on renal outcomes and glycaemic control in T2DM patients with mild CKD and hypertension.

\section{Methods}

A total of 76 patient records ( 38 males and 38 females) of patient ages 40-60 were analysed from the kidney unit at Punjab Care hospital, Lahore, Pakistan. All patients had a confirmed history of T2DM with mild CKD and established hypertension.

Eligible patients were divided into two groups of 38 individuals each. Group SU received gliclazide monotherapy (SU) or Alogliptin (DPP-4i)+gliclazide (SU) add on therapy. All patients were followed up for 12 months.

\section{Results}

The alogliptin (DPP-4i) plus gliclazide (SU) add on therapy group, in comparison to the group only receiving gliclazide (SU), showed a significant difference in eGFR values. The mean \pm SD GFR values post 12 months were $74.8 \pm 0.31$ (95\% $\mathrm{Cl}: 74.8 \pm 0.09 ; 74.7-74.9)$ and $76.1 \pm 0.25$ (95\% $\mathrm{Cl}$ : $76.1 \pm 0.08 ; 76.0-76.2)$ for SU vs. SU+DPP-4i, respectively, with mean calculated effect size of 1.6,. HbA1c, 1,5 AG and ipid profile values have significantly changed $(p<0.05)$ while blood pressure values showed no change. The mean \pm SD systolic blood pressure readings post 12 months for for SU vs. SU+DPP-4i were $131.4 \pm 10.4(95 \% \mathrm{Cl} 131.4 \pm 3.3 ; 128.1-$ 134.7), and $131.8 \pm 9.9(95 \% \mathrm{Cl} 131.8 \pm 3 ; 128.8-134.8)$, respectively.

\section{Conclusion}

In the present study, patients using alogliptin in addition to sulfonyl urea showed improved glycaemic control and lipid profile without increased occurrence of hypoglycaemia. We concluded that, DPP-4i inhibitors are safe treatment options for patients with type 2 diabetes and mild degree of renal impairment.

\section{Key Words}

Diabetes mellitus, chronic kidney disease, DPP4 inhibitors 


\section{What this study adds:}

\section{What is known about this subject?}

Diabetes and hypertension are both listed as major causes of renal complications. Many clinical studies have debated that DPP-4i can offer some degree of nephroprotection.

\section{What new information is offered in this study?}

The Alogliptin plus gliclazide add on therapy group, showed a significant difference in the renal outcomes values beyond glucose-control.

\section{What are the implications for research, policy, or} practice?

Further studies are needed so that these data can help guide clinicians towards selecting appropriate antidiabetic agents to treat T2DM with chronic renal disease.

\section{Background}

Type 2 diabetes mellitus (T2DM) individuals are more prone to microvascular complications such as (CKD) that is associated with increased morbidity, mortality and progression to end-stage renal disease. ${ }^{1}$ Diabetes and hypertension are listed as major causes of CKD which is particularly important in developing countries such as Pakistan. $^{2}$ Up to 80 per cent of CKD patients have hypertension which significantly contributes to the progression of kidney disease toward end stage renal disease (ESRD). The National Kidney Foundation clinical practice guidelines (NKF-KDOQI) recommended a blood pressure goal of $<130 \mathrm{mmHg}$ systolic and $<80 \mathrm{mmHg}$ diastolic for all CKD patients. ${ }^{3}$ Proteinuria is calculated by albuminto-creatinine ratio (ACR) and protein-to-creatinine ratio (PCR) is used to predict renal disease progression. ${ }^{4}$

Anti-hyperglycemic treatment options for CKD patients with T2DM are limited because of the safety and tolerability concerns such as metformin being contraindicated in patients with creatinine clearance $<60 \mathrm{~mL} / \mathrm{min}^{5-8}$

Dipeptidyl peptidase 4 inhibitors (DPP4i) are well-tolerated treatment options for T2DM patients with any degree of renal impairment. ${ }^{9-12}$ These agents lower blood glucose by inhibiting the DPP-4 enzyme that controls GLP-1 and glucose-dependent insulinotropic polypeptide (GIP) degradation. These hormones improve the control of glucose-dependent secretion of insulin and suppress glucagon secretion as shown in Figure 1.

Dipetidyl peptidase enzymes limit the action of these chemicals and lead to rapid inactivation ${ }^{13}$ Studies have suggested some links between obesity and an increased expression of DPP-4 enzymes which highlights the pathophysiological role which these enzymes can play in diabetes. ${ }^{14-15}$ This is a desirable effect when insulin secretion and glycaemic control is required ${ }^{16}$ This class of drugs includes sitagliptin, vildagliptin, saxagliptin, linagliptin and alogliptin.

Measuring glomerular filtration rate (GFR) is recommended by the National Kidney Foundation using prediction equations such as (CKD) Epidemiology Collaboration (CKDEPI) equation and the Modification of Diet in Renal Disease (MDRD) equation. ${ }^{17-19}$ Renal function is moderately decreased if GFR is $<60 \mathrm{ml} \cdot \mathrm{min}^{-1} .1 .73 \mathrm{~m}^{-2}$ and considered severely decreased if GFR is $<30 \mathrm{ml} \cdot \mathrm{min}^{-1} \cdot 1.73 \mathrm{~m}^{2}$ ). Based on the above classification, Grade 2 patients are those who have mildly reduced GFR values that range between (60-89 $\left.\mathrm{ml} \cdot \mathrm{min}^{-1} \cdot 1.73 \mathrm{~m}^{-2}\right){ }^{20}$

Clinical studies have suggested that DPP-4i can offer some degree of nephroprotection. Previous studies using saxagliptin and linagliptin suggested an involvement of DPP4 in nephroprotection. This appears to be via a pathway independent of glycaemic control, such as the improvement of albuminuria. ${ }^{21}$

Alogliptin is a DPP-4i that is used in T2DM treatment. ${ }^{22}$ There is a dearth of studies that specifically compare DPP-4i usage in comparison to other anti-diabetic drugs for CKD and hypertensive patients. CKD In patients with eGFR $\geq 60 \mathrm{~mL} / \mathrm{min}$ there is no need for dose adjustment of alogliptin but in patients with eGFR $30-60$ and $<30 \mathrm{~mL} / \mathrm{min}$ it is reduced to $12.5 \mathrm{mg}$ and $6.25 \mathrm{mg} /$ daily respectively. ${ }^{23-24}$

Glycaemic control can be evaluated by analysing long term markers such as $\mathrm{HbA1c}$ and short term markers such as 1,5anhydroglucitol (1,5-AG). ${ }^{25}$ In patients with kidney disease, the levels of 1,5 AG are overall stable and is used in our hospital as a short glycemic marker. Thus, 1,5 AG of patients were recorded in this study. ${ }^{26}$

The aim of the present study was to retrospectively evaluate T2DM with established mild CKD (eGFR 60$89 \mathrm{~mL} / \mathrm{min} / 1.73 \mathrm{~m}^{2}$ ) and hypertension. Patient records were divided into two treatment groups; Group SU who received only SU (gliclazide) and Group SU+DPP-4i who received alogliptin in combination with gliclazide. Eligible patients were assessed for the levels of glycated hemoglobin (HbA1c), fasting blood glucose (FBG), 1,5-AG, eGFR and post prandial blood glucose (PPBG) for 12 months. 


\section{Method}

This was a retrospective observational exploratory study performed in patients attending the Renal Unit at Punjab Care hospital, Lahore, Pakistan from February 2014 till April 2015. The study protocol was approved by the Ethics Committee of Punjab Care hospital, Lahore, Pakistan (IP45603). The study was performed in accordance with Good Clinical Practice standards and the ethical principles that have their origin in the Declaration of Helsinki.

The selection criteria for the study population were as follows. All patients had a confirmed history ofT2DM which was evident by blood glucose and $\mathrm{HbA} 1 \mathrm{c}$ levels $(6.5-8.0 \%$ at screening). The mean $\pm S D$ estimated duration of diabetes in

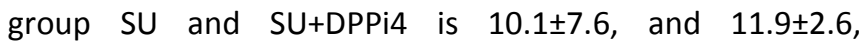
respectively. All patients had established CKD stage 1 (eGFR $60-89 \mathrm{ml} / \mathrm{min} / 1.73 \mathrm{~m}^{2}$; using the Modification of Diet in Renal Disease equation) and a history of established hypertension as evident from patient medical reports were included.

Patients who were on dialysis or likely to require dialysis during the study duration were excluded. All patients had been diagnosed with mild CKD based on the presence of proteinuria evidenced by $A C R>2.5 / 3.5 \mathrm{mg} / \mathrm{mmol}$ and $P C R>15 \mathrm{mg} / \mathrm{mmol}$. Patients on insulin treatment or have received insulin within 12 weeks prior to screening, and patients with type 1 DM were excluded. In addition, patients with a history of ketoacidosis, acute renal disease, renal transplant, presence of liver disease, a cardiovascular event within 12 weeks, pregnancy and those who had received DPP-4i therapy within the previous 12 weeks = were also excluded.

Besides, patients with triglcyerides $>600 \mathrm{mg} / \mathrm{dL}$ were also excluded from the study.

The study population consisted of a total of 76 patients ( 38 males and 38 females) of age range 40-60 years who visited the hospital from February 2014 till April 2015.

Patient records were examined in detail for information such as any medical history, smoking habits, body mass index and physical examinations. Laboratory test records for the following parameters; fasting blood glucose (FBG), HbA1c levels, lipid profile and eGFR were recorded.

Selected diabetic patients with CKD and hypertension were divided into two groups. Each group had 38 individuals. Each patient either received gliclazide monotherapy (Group $\mathrm{SU}$ ), or Alogliptin (DPP-4i) plus gliclazide (Group SU+DDP4i) add on therapy. Outpatient visits were scheduled at 3, 6, and 12 months during the first year of the study. All patients were followed up for 12 months for any arising renal complication.

Group SU received an oral dose of twice daily $60 \mathrm{mg}$ gliclazide, a (SU) monotherapy (Diamicron ${ }^{\circledR}$ MR 60mg; Servier, Pakistan) as prescribed by the patient's physician. According to the American Society's recommendations $60 \mathrm{mg}$ gliclazide would adjust the level of $\mathrm{HbA} 1 \mathrm{c}$ to $\leq 6.5 \%$. Group SU+DPP4i had received an add on therapy of a twice daily dose of $60 \mathrm{mg}$ gliclazide (SU) (Diamicron $^{\circledR}$ MR 60 tablets; Servier, Pakistan) plus an oral once daily $12.5 \mathrm{mg}$ dose of Alogliptin; a DPP-4i (Nesina 25mg; Takeda, Thailand).

During treatment and follow-up periods, patients had received standard medications for CKD and antihyperlipidemic drugs that are listed in Table 1. Replacement or altered dosage of anti-hyperlipidemic drugs was not observed.

During the first year of the observational study, outpatient visit records at 3,6 , and 12 months were examined in detail. All patients had been followed up for renal complications. At each outpatient visit patients' glycated hemoglobin (HbA1c), fasting blood glucose (FBG), blood pressure, serum creatinine, 1,5 AG levels, urea, electrolytes and eGFR were obtained from the patients' medical records.

\section{Statistical analysis}

Package for Social Science (SPSS) Version 21 (SPSS Inc., Chicago IL, USA) was used for statistical analysis. The two treatment groups were compared using paired t-test and the calculated $p$-value was considered to be significant if it was $\leq 0.05$. The effect size has been calculated for mean $\pm S D$ GFR values.

\section{Results}

A total of 76 patients ( 38 males and 38 females) of the age range 40-65 were selected. Patient records were divided into two treatment groups.

Group SU had 38 individuals (19 females and 19 males) with mean $\pm S D$ age $(\mathrm{yr})$ and weight $(\mathrm{kg})$ of $62.9 \pm 6.1$, and $75.2 \pm 4.1$, respectively. Group SU+DPP-4i had 38 patients (19 females and 19 males) with mean \pm SD age and weight of $58.9 \pm 7$ and $85.5 \pm 75.1$ respectively. The baseline demographic and clinical characteristics of both groups are provided in Table 1. No statistical significance was noted in all baseline characteristics. 


\section{Glycemic control}

Initially, the diabetes profile of the patients in Group SU and Group SU+DPP-4i showed no significant difference in $\mathrm{HbA1C}$ and FBG levels prior to treatment administration as represented in Figures 2 and 3, respectively.

There was a significant difference in $\mathrm{HbA} 1 \mathrm{c}$ and FBG between both groups after treatment administration as presented in Table 2.

The levels of HbA1c, FBG and PPBG in Group SU+DPP-4i were significantly lower $(p<0.05)$ when compared to Group $\mathrm{SU}$ (Figures 1-3). As shown in Figure 3 the decrease in FBG was $57.1 \pm 16.8,67.35 \pm 20.6 \mathrm{mg} / \mathrm{dl}$ for Group SU and Group SU+DPP4i respectively. As shown in Figure 2, the mean \pm SD decrease from baseline in $\mathrm{HbA1c}$ for Group SU and Group SU+DPP-4i were $1.0 \pm 0.48$ and $2.06 \pm 0.19 \%$., respectively. In Figure 3 the decrease in PPBG was 5.1 \pm 3.6 and $16.35 \pm 0.1 \mathrm{mg} / \mathrm{dl}$ for Group SU and Group SU+DPP4i respectively.

As shown in Figure 4, comparing 1,5 AG $(\mu \mathrm{g} / \mathrm{mL})$ values at baseline and post 3, 6 and 12 months of treatment $(n=76)$ between Group SU and Group SU+DDP-4i approached a significant difference at $(p<0.001) 3$ months and 12 months post-treatment but not at 6 months. Overall, the level of 1,5 AG was significantly higher in the Group SU+DPP-4i compared to SU group.

\section{Lipid control}

As shown in Table 2, comparing the lipid profile (LDL, HDL and TG) of individuals in Group SU and Group SU+DDP-4i showed significant difference $(p<0.05)$ at baseline, 3 months and 6 months. However it normalized 12 months posttreatment.

\section{Renal outcomes}

The ACR and PCR values $(\mathrm{mg} / \mathrm{mmol})$ showed a mild nonsignificant decrease in both Groups. The mean $\pm S D$ decrease from baseline in ACR and PCR for Group SU and Group SU+DPP-4i were $2.1 \pm 1.2$ and $1.7 \pm 0.6 \mathrm{mg} / \mathrm{mmol}$, respectively.

As seen in Figure 5, the eGFR values in the Group SU+DPP-4i compared to Group SU were significantly increased $(p<0.05)$ at 6 months, 9 months and 12 months post-treatment.

The mean $\pm S D$ decrease from baseline in ACR and PCR for Group SU and Group SU+DPP-4i were respectively $0.1 \pm 0.13$ and $0.8 \pm 0.3 \mathrm{ml} \cdot \mathrm{min}^{-1} \cdot 1.73 \mathrm{~m}^{-2}$

Estimated mean \pm SD GFR values were $75.1 \pm 0.22,74.9 \pm 0.12$
$74.8 \pm 0.31$ and were $76.1 \pm 0.35,76.5 \pm 0.25,76.1 \pm 0.25$ for SU vs. SU+DPP-4i, with calculated effect size of $0.63,0.77$, and 1.6 post 6,9 and 12 months respectively.

The mean SD data and statistical analysis values have been summarized in Table 2. All values are in Mean \pm SD. In terms of hypoglycaemia, there were two hypoglycaemic events reported in a total of two patients (one events in each patient) (2.5\%).

\section{Discussion}

T2DM patients are often plagued by microvascular complications such as CKD and yet there is a lack of studies about the effects of DPP- $4 \mathrm{i}$ in such patients.

In the present study we assessed the effect of alogliptin (DPP-4i) plus gliclazide (SU) in patients with T2DM, mild CKD and hypertension compared to patients who only received gliclazide (SU).

\section{Glycaemic control and hypoglycaemic episodes}

The present study showed that using alogliptin as add on therapy to gliclazide caused a significant $(p<0.05)$ reduction in $\mathrm{HbA1c}$ and FBG levels when compared to Group SU monotherapy. This is in line with other studies that have shown better glycaemic control when adding DPP-4i therapy to metformin, insulin or sulfonylureas. ${ }^{27}$

In the present study the level of 1,5-AG was measured as a short term marker for glycemic control since it has been found to be a more sensitive tool to check day to day glycemic control. ${ }^{28}$ The 1,5-AG values and postprandial glucose levels showed an overall decrease and this tendency toward improvement suggested the possibility that alogliptin might improve postprandial hyperglycaemia. Speculation that DPP-4 inhibitors would improve blood glucose variability by suppressing postprandial hyperglycaemia requires more extensive studies to explore this aspect.

In fact 1,5-AG levels have also been linked to renal damage induced hyperglycaemia, where the increase in renal damage in diabetes patients was found to be more strongly related to 1,5-AG, than to FBG or HbA1C levels. In our study, 1,5-AG, appeared to be an attractive marker for not only detecting glycaemia severity but also for detecting renal damage induced hyperglycemia. ${ }^{29}$ In both treatment groups, there were minor differences in hypoglycaemia incidences. This difference was not significant in our results but previous studies have shown that sulfonyl urea treated patients can often experience significant hypoglycaemic 
episodes. ${ }^{30}$ Thus SU drugs should be used with caution at the lowest effective dosage and ongoing glucose level monitoring has been recommended for patients on this therapy regimen. However, DPP-4i treatment regimens have not been linked to significant hypoglycaemic episodes and this could be due to its effect on glucagon suppression during meal consumption and persistent glucagon suppression during hypoglycemia. ${ }^{31}$ Thus, alogliption use may offer a potential add on therapy for patients diagnosed with other type of diabetes such as type 1 without significantly increasing the risk of hypoglycaemic episodes.

\section{Blood pressure, lipid profile and BMI}

Our results showed a significant improvement in the lipid profile (HDL, LDL and TG) when alogliptin was used in Group SU+DPP-4i in time points three, six, nine and twelve months. This suggests using alogliptin can improve the lipid profile of T2DM patients. This is of particular importance when considering an increased cardiovascular complication risk in T2DM patients. ${ }^{32}$ Our results agree with a previous study that showed improvement in lipid profile when using alogliptin. ${ }^{33}$ Mechanisms to explain this effect include GLP-1 induced myocardial protection, anti-inflammatory role by reduction of $C$ reactive protein levels and enhancing endothelial function. ${ }^{34}$ However, lipid profiles were not significant at the 12 month time point which suggests that other measures need to be added later on during the treatment course of dyslipidaemia.

Our participants' blood pressure (BP) values were less than $125 \mathrm{mmHg}$ which is in line to the NKF guidelines for CKD. The blood pressure values of patients in Group SU did not show any significant difference when compared to Group SU+DDP4 $\mathrm{i}$ although there was a trend for lower values in patients receiving alogliptin. This is likely to be due to an increase in sodium excretion which leads to a reduction in blood volume. The latter suggests a renal protection property associated with the use of alogliptin. ${ }^{35}$

Patients' weight measurements were statistically significant when Group SU and Group SU+DPP-4i were compared. There was a significant increase in the weight of patients belonging to the Group SU but not in the Group SU+DPP-4i. Theories about how weight gain is prevented in DPP-4i include explanations of enhanced postprandial lipid mobilization and oxidation by alogliptin. ${ }^{36}$ This might be due to an increase in the postprandial thermogenesis. In the long term, the avoidance of weight gain can have indirect effects on cardiovascular risks including blood pressure, insulin resistance and cholesterol levels.

\section{Renal function}

A point of interest in the present study was to assess if using DPP-4i had a deleterious effect on renal function by estimating eGFR values in patients having mild renal disease. There was a significant $(p<0.05)$ difference between the eGFR values in both groups with improved eGFR values in Group SU+DPP-4i. Previous studies have shown similar results. $^{31}$ Previous studies have shown a significant reduction of ACR values after 24 weeks post treatment with linagliptin. Nevertheless, $\mathrm{HbA}_{\mathrm{C}}$ values did not change and this suggested that the effect was likely to be independent of glycaemic control. ${ }^{37}$

Although our study did not show a significant change in ACR or PCR values post DPP4i treatment, however eGFR improvement might suggest possible nephroprotective effects. The DPP4i exact nephroprotective role is still debated and has not been fully elucidated yet. However, a possible mechanism may include an improvement in the variability of blood glucose which is well known in inhibiting the progress of diabetes complications. ${ }^{38}$

Studies on the effect of DDP4i, including animal studies, have suggested improved eGFR values, modulation of nephropathy and reduction of albumin levels independent of glycaemic control. ${ }^{39}$

Furthermore, it has been proposed by in vitro data that glucagon-like peptide-1 (GLP-1) receptor expression in the kidneys is high. This makes the kidney a feasible organ for DPP-4 activity and a target in the extra-pancreatic effects of incretins. It has also been shown that upregulation of DPP4 expression in the renal glomeruli during inflammation, contributed to the development of diabetes-induced glomerulosclerosis. Studies have shown that using DPP-4i increased the local levels of GLP-1 in the kidneys which promoted anti-inflammation and anti-oxidation. ${ }^{40}$

Indeed, if future larger prospective clinical studies could confirm DPP4i nephroprotective role and its ability to reduce albumin levels especially, this would be an important progress in clinical practice involving diabetic CKD patients. ${ }^{4}$

\section{Conclusion}

In summary, it can be concluded that DPP-4 inhibitors are effective and safe treatment options for patients with type 2 diabetes and mild degree of renal impairment. Using alogliptin in such patients as add on treatment to sulfonyl urea, resulted in an improved glycaemic control, and lipid profile that did not increase the chance of hypoglycaemic episodes. The inhibition of DPP-4 requires further 
exploration as a potential strategy in the treatment of diabetic CKD patients. Our data have suggested a possible nephroprotective role for DPP-4i drugs. Preliminary studies have shown that lowering the degree of glycaemia can contribute to other therapies that aim to preserve renal function. In the future consideration can be given to the possibility that DPP-4 inhibitors can be used for nephroprotection although this is only suggested by preliminary observations and requires further studies to explore this strategy. Larger prospective clinical trials designed primarily to test renal outcomes are currently ongoing and could contribute to influence potential future therapeutic choices. Further studies are needed so that these data can help to guide clinicians to choose appropriate anti-diabetic agents to treat T2DM with chronic renal disease.

\section{References}

1. Woo KT, Choong HL, Wong KS, et al. The contribution of chronic kidney disease to the global burden of major non-communicable diseases. Kidney Int. 2011;80(12):1258-1270. doi: 10.1038/ki. 2012.39.

2. Levey AS, Coresh J. Chronic kidney disease. Lancet. 2012;379(9811):165-80. doi: 10.1016/S01406736(11)60178-5.

3. Toto RD. Treatment of hypertension in chronic kidney disease. Semin Nephrol. 2005;25(6):435-9. doi: 10.1016/j.semnephrol.2005.05.016.

4. Gerstein HC, Mann JF, Yi Q, et al. Albuminuria and risk of cardiovascular events, death, and heart failure in diabetic and nondiabetic individuals. JAMA. 2001;286(4):421-6. doi: 10.1001/jama.286.4.421.

5. Rother KI. Diabetes treatment-bridging the divide. $\mathrm{N}$ Engl J Med. 2007;356(15):1499. doi: 10.1056/NEJMp078030

6. Inzucchi SE. Oral antihyperglycemic therapy for type 2 diabetes: scientific review. JAMA. 2002;287(3):360-72. doi: 10.1001/jama.287.3.360.

7. Kidney Disease Outcomes Quality Initiative. Clinical practice guidelines and clinical practice recommendations for diabetes and chronic kidney disease. Am J Kidney Dis. 2007;49(2):S12-154. doi: 10.1053/j.ajkd.2006.12.005.

8. Howse PM, Chibrikova LN, Twells LK, et al. Safety and efficacy of incretin-based therapies in patients with type 2 diabetes mellitus and CKD: A systematic review and meta-analysis. Am J Kidney Dis. 2016. doi: 10.1053/j.ajkd.2016.06.014.

9. Kountz D. The dipeptidyl peptidase (DPP)-4 inhibitors for type 2 diabetes mellitus in challenging patient groups.
Adv Ther. 2013;30(12):1067-85. doi: 10.1007/s12325013-0071-y.

10.Zhong J, Rao X, Rajagopalan S. An emerging role of dipeptidyl peptidase 4 (DPP4) beyond glucose control: potential implications in cardiovascular disease. Atherosclerosis. 2013;226(2):305-14. doi: 10.1016/j.atherosclerosis.2012.09.012.

11. Blüher M. Adipokines-removing road blocks to obesity and diabetes therapy. Mole Met. 2014;3(3):230-40. doi: 10.1016/j.molmet.2014.01.005.

12. Kim W, Egan JM. The role of incretins in glucose homeostasis and diabetes treatment. Pharmacol Rev. 2008;60(4):470-512. doi: 10.1124/pr.108.000604.

13. Turner R, Holman R, Cull C, et al. Intensive blood-glucose control with sulphonylureas or insulin compared with conventional treatment and risk of complications in patients with type 2 diabetes (UKPDS 33). UK Prospective Diabetes Study (UKPDS) group. Lancet. 1998;352(9131):837-53. http://doi.org/10.1016/s01406736(98)07037-8.

14. Ramirez G, Morrison AD, Bittle PA. Clinical practice considerations and review of the literature for the Use of DPP-4 inhibitors in patients with type 2 diabetes and chronic kidney disease. Endocr Pract. 2013;19(6):102534. doi: 10.4158/EP12306.RA

15. Russo E, Penno G, Del Prato S. Managing diabetic patients with moderate or severe renal impairment using DPP-4 inhibitors: focus on vildagliptin. Diabetes Metab Syndr Obes. 2013;6:161-70. doi: 10.2147/DMSO.S28951

16. Scheen AJ. Pharmacokinetics and clinical use of incretinbased therapies in patients with chronic kidney disease and type 2 diabetes. Clin Pharmacokinet. 2015;54(1):121. doi: 10.1007/s40262-014-0198-2.

17. Levey AS, Stevens LA, Schmid CH, et al. A new equation to estimate glomerular filtration rate. Ann Intern Med. 2009;150(9):604-12.

https://www.ncbi.nlm.nih.gov/pubmed/19414839.

18. Rigalleau V, Lasseur C, Perlemoine C, et al. Estimation of glomerular filtration rate in Diabetes Care diabetic subjects: Cockcroft formula or modification of Diet in Renal Disease study equation?. Diabetes Care. 2005;28(4):838-43. https://doi.org/10.2337/diacare.28.4.838

19. Levey AS, Coresh J, Greene T, et al. Using standardized serum creatinine values in the modification of diet in renal disease study equation for estimating glomerular filtration rate. Ann Intern Med. 2006;145(4):247-54. doi: https://www.ncbi.nlm.nih.gov/pubmed/16908915.

20. The Renal Association.. CKD stages. 2013 [Available from: http://www.renal.org/information-resources/the- 
uk-eckd-guide/ckd-stages\#sthash.nbCheSAD.dpbs (accessed 12 february 2018)

21. Penno G, Garofolo M, Del Prato S. Dipeptidyl peptidase4 inhibition in chronic kidney disease and potential for protection against diabetes-related renal injury. Nutr Metab Cardiovasc Dis. 2016;26(5):361-73. doi: 10.1016/j.numecd.2016.01.001

22. Cahn A, Cernea S, Razl. An update on DPP-4 inhibitors in the management of type 2 diabetes. Expert Opin Emerg Drugs. 2016;21(4):409-19. doi: 10.1080/14728214.2016.1257608.

23. Arnouts P, Bolignano D, Nistor I, et al. Glucose-lowering drugs in patients with chronic kidney disease: a narrative review on pharmacokinetic properties. Nephrol Dial Transplant. 2014;29(7):1284-300. doi: 10.1093/ndt/gft462.

24. Ioannidis I. Diabetes treatment in patients with renal disease: Is the landscape clear enough?. World J Diabetes. 2014;5(5):651-8. doi: 10.4239/wjd.v5.i5.651.

25. Buse JB, Freeman JL, Edelman SV, et al. Serum 1,5anhydroglucitol (GlycoMark): a short-term glycemic marker. Diabetes Technol Ther. 2003;5(3):355-63. doi:.org/10.1089/152091503765691839.

26. Emoto M, Tabata $T$, Inoue $T$, et al. Plasma 1,5anhydroglucitol concentration in patients with end-stage renal disease with and without diabetes mellitus. Nephron. 1992;61(2):181-6. doi:10.1159/000186868

27. Kusunoki M, Sato D, Nakamura T, et al. The Beneficial Effects of the DPP-4 Inhibitor Alogliptin on Hemoglobin A1c and Serum Lipids in Japanese Patients with Type 2 Diabetes. Drug Res. 2016;66(1):18-22. doi: 10.1055/s0035-1547254.

28. Dungan KM. 1,5-anhydroglucitol (GlycoMark) as a marker of short-term glycemic control and glycemic excursions. Expert Rev Mol Diagn. 2008;8(1):9-19. doi: 10.1586/14737159.8.1.9.

29. Yamanouchi T, Kawasaki T, Yoshimura T, et al. Relationship between serum 1,5-anhydroglucitol and urinary excretion of $\mathrm{N}$-acetylglucosaminidase and albumin determined at onset of NIDDM with 3-year follow-up. Diabetes Care. 1998;21(4):619-24. doi: https://www.ncbi.nlm.nih.gov/pubmed/9571353.

30. Eriksson JW, Bodegard J, Nathanson D, et al A. Sulphonylurea compared to DPP-4 inhibitors in combination with metformin carries increased risk of severe hypoglycemia, cardiovascular events, and allcause mortality. Diabetes Res Clin Pract. 2016;117:3947. doi: 10.1016/j.diabres.2016.04.055.

31. Del Prato S, Fleck $P$, Wilson $C$, et al. Comparison of alogliptin and glipizide for composite endpoint of glycated haemoglobin reduction, no hypoglycaemia and no weight gain in type 2 diabetes mellitus. Diabetes Obes Metab. 2016;18(6):623-7. doi: 10.1111/dom.12643.

32. Eliasson B, Möller-Goede D, Eeg-Olofsson K, et al. Lowering of postprandial lipids in individuals with type 2 diabetes treated with alogliptin and/or pioglitazone: a randomised double-blind placebo-controlled study. Diabetologia. 2012;55(4):915-25. doi: 10.1007/s00125011-2447-3.

33. Khan FZ, Heck PM, Hoole SP, et al. DPP-4 inhibition by sitagliptin improves the myocardial response to dobutamine stress and mitigates stunning in a pilot study of patients with coronary artery disease. Circ Cardiovas Imaging. 2010;3(2):195-201. doi: 10.1161/CIRCIMAGING.109.899377

34. Duvnjak L, Blaslov K. Dipeptidyl peptidase-4 inhibitors improve arterial stiffness, blood pressure, lipid profile and inflammation parameters in patients with type 2 diabetes mellitus. Diabetol Metab Syndr. 2016;8(1):1. doi: 10.1186/s13098-016-0144-6.

35. Hocher B, Reichetzeder C, Alter ML. Renal and cardiac effects of DPP4 inhibitors--from preclinical development to clinical research. Kidney Blood Press Res. 2012;36(1):65-84. doi: 10.1159/000339028.

36. Groop PH, Cooper ME, Perkovic V, et al. Linagliptin lowers albuminuria on top of recommended standard treatment in patients with type 2 diabetes and renal dysfunction. Diabetes Care. 2013;36(11):3460-8. doi: 10.2337/dc13-0323.

37. Hattori S. Sitagliptin reduces albuminuria in patients with type 2 diabetes. Endocr J. 2011;58(1):69-73. URL: https://www.ncbi.nlm.nih.gov/pubmed/21206136.

38. Gorriz JL, Nieto J, Navarro-Gonzalez JF, et al. Nephroprotection by hypoglycemic agents: Do we have supporting data? J Clin Med. 2015;4(10):1866-89. doi: 10.3390/jcm4101866.

39. Panchapakesan U, Mather A, Pollock C. Role of GLP-1 and DPP-4 in diabetic nephropathy and cardiovascular disease. Clin Sci. 2013;124(1):17-26. doi: 10.1042/CS20120167.

40. Kodera R, Shikata K, Kataoka HU, et al. Glucagon-like peptide-1 receptor agonist ameliorates renal injury through its anti-inflammatory action without lowering blood glucose level in a rat model of type 1 diabetes. Diabetologia. 2011;54(4):965-78. doi: 10.1007/s00125010-2028-x.

\section{PEER REVIEW}

Not commissioned. Externally peer reviewed. 


\section{CONFLICTS OF INTEREST}

The authors declare that they have no competing interests.

\section{ETHICS COMMITTEE APPROVAL}

Ethics Committee of Punjab Care hospital, Lahore, Pakistan

[IP-45603]

Figure 1: Mechanism of action of Dipeptidyl peptidase-4 (DPP-4) inhibitors

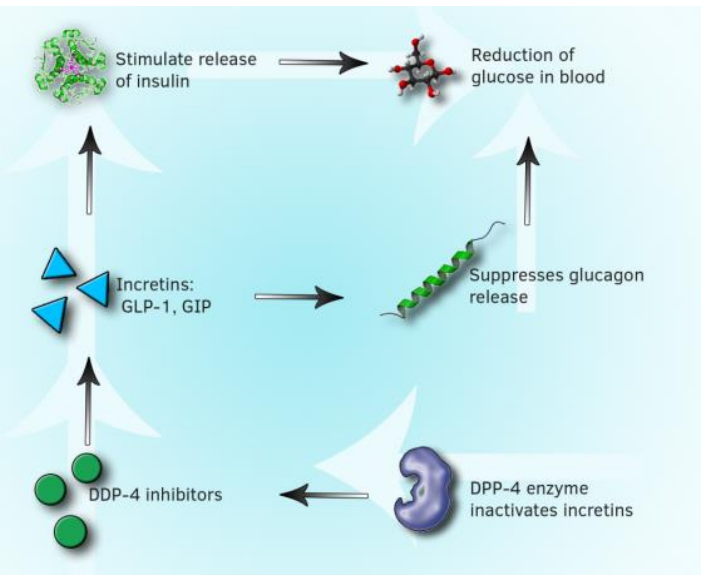

Figure 2: The effect of sulfonylurea (Group SU, blue line) and sulfonylurea+DDP4i (Group SU+DDP4i, red line) on patients over the 12 months observation period. Each point represents the mean \pm SD glycated hemoglobin levels (HbA1c \%) at baseline and post $3,6,9$ and 12 months of treatment, $(n=76) .{ }^{*} p<0.05$ was taken as significant difference compared to the baseline data

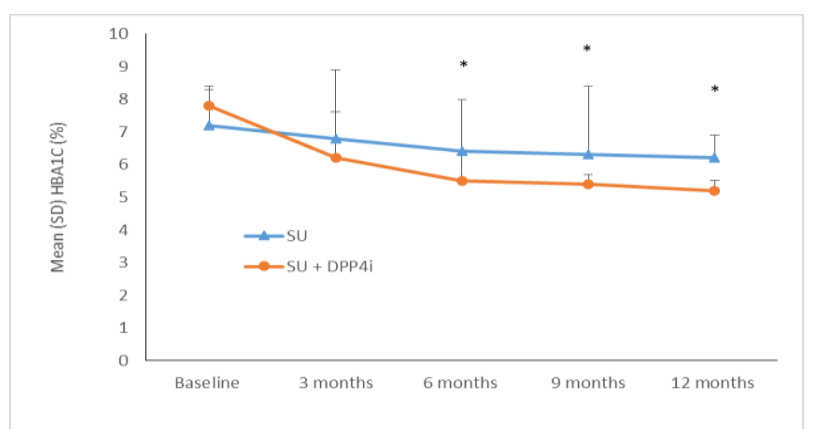

Figure 3: The effect of sulfonylurea (Group SU, blue line) and sulfonylurea+DDP4i (Group SU+DDP4i, red line) on patients over the 12 months observation period. Each point represents the mean \pm SD fasting blood glucose levels $(\mathrm{mg} / \mathrm{dL}$ ) at baseline and post $3,6,9$ and 12 months of treatment, $(n=76){ }^{*} p<0.05$ was taken as significant difference compared to the baseline data

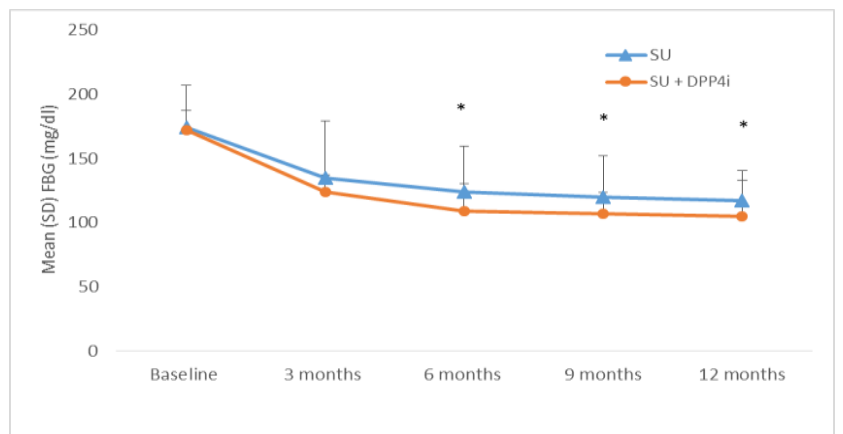


Figure 4: The effect of sulfonylurea (Group SU, blue line) and sulfonylurea+DDP4i (Group SU+DDP4i, red line) on patients over the 12 months observation period. Each point represents the mean \pm SD 1,5 AG $(\mu \mathrm{g} / \mathrm{mL})$ values at baseline and post 3 , 6,9 and 12 months of treatment $(n=76) .{ }^{*} p<0.05$ was taken as significant difference compared to the baseline data

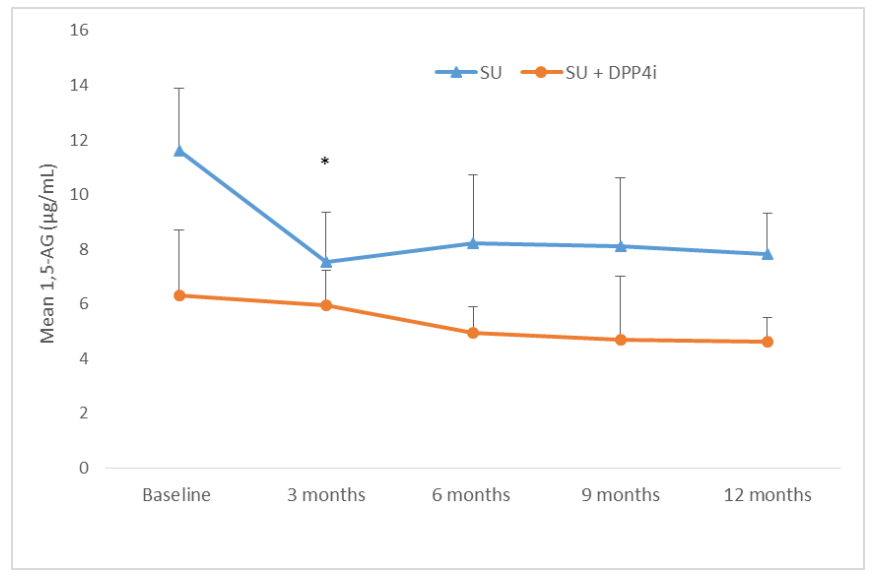

Figure 5: The effect of sulfonylurea (Group SU, blue line) and sulfonylurea+DDP4i (Group SU+DDP4i, red line) on patients over the 12 months observation period. Each point represents the Mean \pm SD eGFR $(\mathrm{ml} / \mathrm{min})$ values calculated at baseline and post 3, 6, 9 and 12 months of treatment $(n=76){ }^{*} p<0.05$ was taken as significant difference compared to the baseline data. Group 1 (blue line): Group SU and Group 2 (red line): Group SU+DPP4i

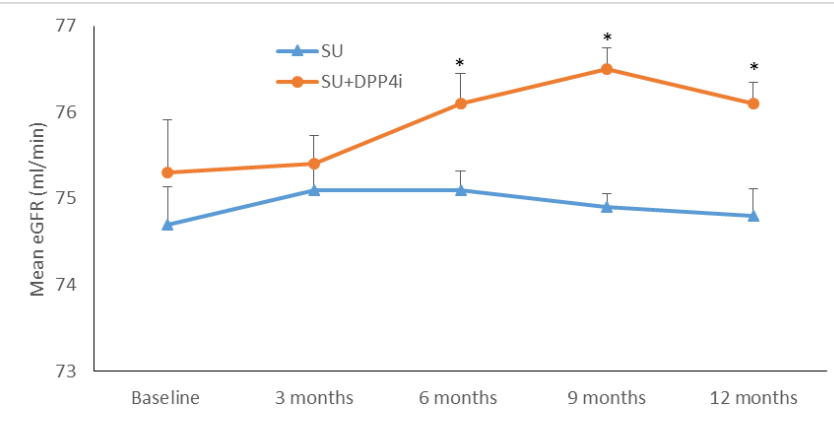

Table 1: Baseline Characteristics of the study Patients $(n=76)$. Data presented in mean \pm SD or $n(\%)$

\begin{tabular}{|c|c|c|c|c|}
\hline \multicolumn{2}{|c|}{$\begin{array}{l}\text { Demographic details } \\
\text { Mean } \pm \text { SD or No (\%) }\end{array}$} & $\begin{array}{l}\text { Group SU (n=38) } \\
\text { Gliclazide (SU) } \\
\text { Monotherapy }\end{array}$ & $\begin{array}{l}\text { Group SU+DPP-4i } \\
\text { ( } n=38) \\
\text { Gliclazide+Alogliptin } \\
\text { (SU+DPP4i) } \\
\text { Add on therapy }\end{array}$ & $P$ values \\
\hline \multicolumn{2}{|l|}{ Age (yr) } & $58.3 \pm 6.1$ & $58.7 \pm 7.1$ & 0.75 \\
\hline \multirow{2}{*}{ Gender (no.) } & Males & $19(50)$ & $19(50)$ & 0.65 \\
\hline & Females & $19(50)$ & $19(50)$ & 0.9 \\
\hline \multirow{2}{*}{ Weight (kg) } & Male & $75.2 \pm 4.2$ & $85.5 \pm 5.1$ & 0.99 \\
\hline & Female & $62.9 \pm 6.1$ & $58.9 \pm 7.0$ & 0.86 \\
\hline $\mathrm{BMI}\left(\mathrm{kg} / \mathrm{m}^{2}\right)$ & BMI & $23.6 \pm 5.6 \mathrm{~kg} / \mathrm{m}^{2}$ & $25.4 \pm 5.2$ & 0.8 \\
\hline \multirow{2}{*}{ Smoking } & Smokers & $03 \pm 2.1$ & $05 \pm 2.3$ & 0.99 \\
\hline & Non-smoking & $35 \pm 13.3$ & $33 \pm 16.7$ & 0.95 \\
\hline CKD status & eGFR (ml. $\left.\mathrm{min}^{-1} \cdot 1.73 \mathrm{~m}^{-2}\right)$ & $74.7 \pm 0.44$ & $75.3 \pm 0.61$ & 0.94 \\
\hline
\end{tabular}




\begin{tabular}{|c|c|c|c|c|}
\hline & Serum creatinine $(\mathrm{mg} / \mathrm{dL})$ & $2.4 \pm 0.7$ & $2.2 \pm 0.5$ & 0.77 \\
\hline & Serum urea $(\mathrm{mmol} / \mathrm{L})$ & $8.5 \pm 1.2$ & $8.7 \pm 1.0$ & 0.55 \\
\hline & $\mathrm{ACR}(\mathrm{mg} / \mathrm{mmol})$ & $12 \pm 2.2$ & $14 \pm 1.6$ & 0.09 \\
\hline & PCR (mg/mmol) & $24 \pm 3.2$ & $21 \pm 1.2$ & 0.91 \\
\hline \multirow{2}{*}{ Blood pressure } & Systolic (mmHg) & $115 \pm 21.9$ & $122 \pm 21.1$ & 0.44 \\
\hline & Diastolic (mmHg) & $80 \pm 18.1$ & $78 \pm 19.8$ & 0.3 \\
\hline Family history & & $4(11.8)$ & $5(5.2)$ & 0.55 \\
\hline Alcohol consumption & & $01(1.3)$ & $2(2.6)$ & 0.43 \\
\hline \multirow{2}{*}{ Diabetes complications } & Diabetes retinopathy & 06 (15.6) & $8(21.5)$ & 0.8 \\
\hline & Diabetes neuropathy & $12(4.5)$ & $10(3.8)$ & 0.9 \\
\hline \multirow{4}{*}{ Medical history } & $\begin{array}{l}\text { Cerebrovascular accident (cerebral } \\
\text { infarction/cerebral haemorrhage) }\end{array}$ & $1(2.6)$ & $2(5.2)$ & 0.5 \\
\hline & Myocardial infarction & $3(7.8)$ & $1(2.6)$ & 0.54 \\
\hline & Angina & $01(2.6)$ & $02(5.2)$ & 0.44 \\
\hline & Dyslipidemia & $04(10.5)$ & 05 (13.5) & 0.54 \\
\hline \multirow{8}{*}{ Medication use } & ARB & $15(41.3 \%)$ & $18(47.3 \%)$ & 1 \\
\hline & Ca antagonists & $12(31.5 \%)$ & $10(26.3 \%)$ & 0.78 \\
\hline & Diuretics & 30 (78.9\%) & $32(84.2 \%)$ & 0.99 \\
\hline & ACE inhibitors & $28(73.6 \%)$ & $31(81.5 \%)$ & 0.65 \\
\hline & Renin inhibitors & 27 (71.0\%) & $29(76.3 \%)$ & 0.96 \\
\hline & $\alpha$ blockers & $3(7.8 \%)$ & $5(13.1 \%)$ & 0.43 \\
\hline & $\beta$ blockers & $2(5.2 \%)$ & $3(7.89 \%)$ & 0.55 \\
\hline & Aldosterone blockers & $1(2.63 \%)$ & $1(2.63 \%)$ & 0.92 \\
\hline \multicolumn{2}{|c|}{ Estimated duration of diabetes (years) } & $10.1 \pm 7.6$ & $11.9 \pm 2.6$ & 0.91 \\
\hline
\end{tabular}

Table 2: The Mean $\pm S D$ and statistical comparison values of different glycemic, and renal parameters measured at baseline and post 3, 6, 9, and 12 months treatment of $S U(n=38)$ and SU+DPP-4i $(n=38) * P<0.050 .{ }^{* * P}<0.01 * * * P<0.001$ SD: standard deviation; SE: standard error; NS: Non-significant

\begin{tabular}{|c|c|c|c|c|}
\hline $\begin{array}{ll}\text { Clinical } & \text { Parameter } \\
\text { Mean } \pm S D & \end{array}$ & Time point & Group SU $(n=38)$ & Group SU+DPP-4i (n=38) & $P$ values \\
\hline \multirow{5}{*}{ LDL } & Baseline & $135.9 \pm 9.3$ & $130.3 \pm 12.3$ & $0:-N S$ \\
\hline & 3 months & $98.03 \pm 5.7$ & $82 \pm 5.6$ & 3: $0.0451^{*}$ \\
\hline & 6 months & $88.2 \pm 5.8$ & $84.7 \pm 5.8$ & 6: $0.0003^{*}$ \\
\hline & 9 months & $86.2 \pm 5.1$ & $83.8 \pm 1.8$ & 9: $0.0263^{*}$ \\
\hline & 12 months & $80.2 \pm 5.2$ & $81.7 \pm 5.8$ & 12: 0.5926 \\
\hline \multirow[t]{5}{*}{$\mathrm{HDL}$} & Baseline & $35.2 \pm 4.1$ & $39.8 \pm 3.3$ & $0:-N S$ \\
\hline & 3 months & $43.1 \pm 1.2$ & $47.9 \pm 2.5$ & 3: $0.0261^{*}$ \\
\hline & 6 months & $41.8 \pm 7.8$ & $45.5 \pm 3.1$ & 6: $0.045^{*}$ \\
\hline & 9 months & $40.2 \pm 1.5$ & $43.2 \pm 7.1$ & 9: $0.0266^{*}$ \\
\hline & 12 months & $39.8 \pm 7.4$ & $41.5 \pm 3.4$ & $12: 0.789$ \\
\hline \multirow[t]{4}{*}{ TG } & Baseline & $186.03 \pm 10.3$ & $180.5 \pm 9.2$ & $0:-N S$ \\
\hline & 3 months & $139.2 \pm 3.9$ & $128.3 \pm 5.2$ & 3: $0.0341^{*}$ \\
\hline & 6 months & $131.8 \pm 6$ & $129 \pm 5.0$ & 6: $0.050^{*}$ \\
\hline & 9 months & $129.1 \pm 2$ & $123 \pm 4.6$ & 9: $0.048^{*}$ \\
\hline
\end{tabular}




\begin{tabular}{|c|c|c|c|c|}
\hline & 12 months & $128.8 \pm 4$ & $119 \pm 5.1$ & 12: 0.6712 \\
\hline \multirow[t]{5}{*}{ HbA1c (\%) } & Baseline & $7.2 \pm 1.2$ & $7.8 \pm 0.5$ & $0:-N S$ \\
\hline & 3 months & $6.8 \pm 2.1$ & $6.2 \pm 1.4$ & $3:<0.0001 * * *$ \\
\hline & 6 months & $6.4 \pm 1.6$ & $5.5 \pm 0.85$ & $6:<0.0001^{* * *}$ \\
\hline & 9 months & $6.3 \pm 2.1$ & $5.4 \pm 0.25$ & $9:<0.0001 * * *$ \\
\hline & 12 months & $6.2 \pm 0.72$ & $5.2 \pm 0.31$ & $12:<0.0001^{* * *}$ \\
\hline \multirow[t]{5}{*}{$1,5-A G$} & Baseline & $5.11 \pm 0.56$ & $6.81 \pm 0.89$ & $0:-N S$ \\
\hline & 3 months & $5.49 \pm 0.68$ & $6.39 \pm 0.62$ & 3: $0.005^{*}$ \\
\hline & 6 months & $5.15 \pm 0.59$ & $6.91 \pm 0.5$ & $6: 0.006$ \\
\hline & 9 months & $5.46 \pm 1.3$ & $7.2 \pm 1.1$ & 9: 0.051 \\
\hline & 12 months & $5.5 \pm 0.87$ & $7.31 \pm 0.5$ & 12: $0.005^{*}$ \\
\hline \multirow[t]{5}{*}{$\mathrm{FBG}(\mathrm{mg} / \mathrm{dl})$} & Baseline & $174 \pm 32.7$ & $172 \pm 15.1$ & $0:-N S$ \\
\hline & 3 months & $135 \pm 44.1$ & $124 \pm 12.8$ & 3: 0.0951 \\
\hline & 6 months & $124 \pm 35.7$ & $109 \pm 21.4$ & $6:<0.005^{* *}$ \\
\hline & 9 months & $120 \pm 31.7$ & $107 \pm 16.7$ & 9: $0.0091^{* *}$ \\
\hline & 12 months & $117 \pm 15.9$ & $105 \pm 35.7$ & $12: 0.0095^{* *}$ \\
\hline \multirow{5}{*}{$\begin{array}{l}\text { Post Prandial Blood Glucose } \\
\qquad \text { (PPBG) (mg/dl) }\end{array}$} & Baseline & $125 \pm 12.1$ & $128 \pm 12.8$ & $0:-N S$ \\
\hline & 3 months & $135 \pm 10.9$ & $115 \pm 16.7$ & $3: 0.0008^{* * *}$ \\
\hline & 6 months & $128 \pm 14.2$ & $110 \pm 11.2$ & $6: 0.0040^{* *}$ \\
\hline & 9 months & $121 \pm 12.2$ & $111 \pm 10.3$ & 9: $0.0050^{* *}$ \\
\hline & 12 months & $120 \pm 8.5$ & $112 \pm 12.9$ & $12: 0.0048^{* *}$ \\
\hline \multirow[t]{5}{*}{ eGFR } & Baseline & $74.7 \pm 0.44$ & $75.3 \pm 0.61$ & $0:-N S$ \\
\hline & 3 months & $75.1 \pm 0.31$ & $75.4 \pm 0.33$ & 3: 0.15 \\
\hline & 6 months & $75.1 \pm 0.22$ & $76.1 \pm 0.35$ & 6: $0.04 *$ \\
\hline & 9 months & $74.9 \pm 0.12$ & $76.5 \pm 0.25$ & 9: $0.05^{*}$ \\
\hline & 12 months & $74.8 \pm 0.31$ & $76.1 \pm 0.25$ & $12: 0.03^{*}$ \\
\hline \multirow[t]{5}{*}{ ACR $(\mathrm{mg} / \mathrm{mmol})$} & Baseline & $12 \pm 2.3$ & $14 \pm 1.8$ & $0:-N S$ \\
\hline & 3 months & $15 \pm 1.2$ & $11 \pm 3.2$ & 3: 0.1334 \\
\hline & 6 months & $14 \pm 1.2$ & $10 \pm 2.1$ & $6: 0.0623$ \\
\hline & 9 months & $14 \pm 1.6$ & $10 \pm 3.4$ & 9: 0.0712 \\
\hline & 12 months & $13 \pm 1.9$ & $10 \pm 1.1$ & 12: 0.0719 \\
\hline \multirow[t]{5}{*}{ PCR $\mathrm{mg} / \mathrm{mmol}$} & Baseline & $24 \pm 3.2$ & $26 \pm 1.2$ & $0:-N S$ \\
\hline & 3 months & $24 \pm 2.3$ & $23 \pm 1.4$ & 3: 0.1571 \\
\hline & 6 months & $23 \pm 2.1$ & $21 \pm 1.2$ & 6: 0.1522 \\
\hline & 6 months & $22 \pm 2.6$ & $21 \pm 1.3$ & 9: 0.2501 \\
\hline & 12 months & $19 \pm 1.8$ & $18 \pm 4.1$ & 12: 0.0588 \\
\hline \multirow[t]{5}{*}{ Systolic BP } & Baseline & $133 \pm 19.2$ & $132.4 \pm 11$ & $0:-N S$ \\
\hline & 3 months & $133.6 \pm 7$ & $131.5 \pm 8$ & 3: 0.0801 \\
\hline & 6 months & $132.6 \pm 4$ & $132.5 \pm 2$ & $6: 0.7811$ \\
\hline & 9 months & $131.9 \pm 15.4$ & $132.1 \pm 9.5$ & 9: 0.0519 \\
\hline & 12 months & $131.4 \pm 10.4$ & $131.8 \pm 9.9$ & 12: 0.1091 \\
\hline \multirow[t]{5}{*}{ Diastolic BP } & Baseline & $83.1 \pm 10.3$ & $80 \pm 12.3$ & $0:-N S$ \\
\hline & 3 months & $81.7 \pm 3.9$ & $80.4 \pm 6.8$ & 3: 0.2765 \\
\hline & 6 months & $82.2 \pm 7.7$ & $81.1 \pm 12.8$ & $6: 0.2611$ \\
\hline & 9 months & $80.6 \pm 3.2$ & $79.9 \pm 8.7$ & 9: 0.4327 \\
\hline & 12 months & $81.5 \pm 1.2$ & $79.9 \pm 8.7$ & 12: 0.9827 \\
\hline \multirow[t]{5}{*}{ Hypoglycemic episodes } & Baseline & $0(0 \%)$ & $0(0 \%)$ & $0:-N S$ \\
\hline & 3 months & $1(3.3 \%)$ & $1(3.3 \%)$ & 3: 0.345 \\
\hline & 6 months & $2(6.7 \%)$ & $1(3.3 \%)$ & 6: 0.456 \\
\hline & 9 months & $1(3.3 \%)$ & $1(3.3 \%)$ & 9: 0.299 \\
\hline & 12 months & $1(3.3 \%)$ & $0(0 \%)$ & 12: 0.276 \\
\hline \multirow[t]{5}{*}{ BMI $\left(\mathrm{kg} / \mathrm{m}^{2}\right)$} & Baseline & $25.1 \pm 5.2$ & $25.6 \pm 5.6$ & $0:-N S$ \\
\hline & 3 months & $25.6 \pm 2.5$ & $25.3 \pm 1.6$ & 3: 0.0654 \\
\hline & 6 months & $25.8 \pm 4.1$ & $24.9 \pm 2.9$ & 6: 0.0703 \\
\hline & 9 months & $25.3 \pm 3.1$ & $24.3 \pm 1.5$ & 9: 0.5828 \\
\hline & 12 months & $25.2 \pm 1.2$ & $24.5 \pm 0.8$ & $12: 0.3490$ \\
\hline
\end{tabular}

